\title{
Classification-Aided SAR and AIS Data Fusion for Space-Based Maritime Surveillance
}

\author{
Maximilian Rodger (1) and Raffaella Guida *
}

check for updates

Citation: Rodger, M.; Guida, R. Classification-Aided SAR and AIS Data Fusion for Space-Based Maritime Surveillance. Remote Sens. 2021, 13, 104. https://doi.org/ $10.3390 /$ rs13010104

Received: 17 November 2020 Accepted: 23 December 2020 Published: 30 December 2020

Publisher's Note: MDPI stays neutral with regard to jurisdictional clai$\mathrm{ms}$ in published maps and institutional affiliations.

Copyright: (C) 2020 by the authors. Licensee MDPI, Basel, Switzerland. This article is an open access article distributed under the terms and conditions of the Creative Commons Attribution (CC BY) license (https:// creativecommons.org/licenses/by/ $4.0 /)$.
Surrey Space Centre, University of Surrey, Guildford GU2 7XH, UK; m.rodger@surrey.ac.uk

* Correspondence: r.guida@surrey.ac.uk

\begin{abstract}
A wide range of research activities exploit spaceborne Synthetic Aperture Radar (SAR) and Automatic Identification System (AIS) for applications that contribute to maritime safety and security. An important requirement of SAR and AIS data fusion is accurate data association (or correlation), which is the process of linking SAR ship detections and AIS observations considered to be of a common origin. The data association is particularly difficult in dense shipping environments, where ships detected in SAR imagery can be wrongly associated with AIS observations. This often results in an erroneous and/or inaccurate maritime picture. Therefore, a classification-aided data association technique is proposed which uses a transfer learning method to classify ship types in SAR imagery. Specifically, a ship classification model is first trained on AIS data and then transferred to make predictions on SAR ship detections. These predictions are subsequently used in the data association which uses a rank-ordered assignment technique to provide a robust match between the data. Two case studies in the UK are used to evaluate the performance of the classification-aided data association technique based on the types of SAR product used for maritime surveillance: wide-area and large-scale data association in the English Channel and focused data association in the Solent. Results show a high level of correspondence between the data that is robust to dense shipping or high traffic, and the confidence in the data association is improved when using class (i.e., ship type) information.
\end{abstract}

Keywords: synthetic aperture radar (SAR); Sentinel-1; ICEYE-X2; automatic identification system (AIS); data fusion; data association; ship classification; ship detection; maritime surveillance

\section{Introduction}

The use of spaceborne Synthetic Aperture Radar (SAR) and Automatic Identification System (AIS) for space-based maritime surveillance has recently seen significant interest on an international scale. The primary advantages of SAR include its wide-area coverage and day and night all-weather observation capabilities. In satellite SAR imagery, objects on the sea surface such as ships and offshore platforms are detected as a collection of bright pixels on a dark background. The AIS is a radio-based communications system that exchanges information (e.g., the ship's identity, type, dimensions, position, speed, etc.) between ships and coastal stations [1]. Recently, advancements in AIS technology have also meant that shipborne AIS signals are now reliably received by satellites in low-Earth orbit (Satellite-AIS or Sat-AIS), thereby extending its surveillance capability to the open ocean and beyond the range of terrestrial-based networks. The data fusion of SAR and AIS allows the observations from each sensor to be combined to provide an effective understanding of maritime activities at sea.

The data association of SAR and AIS (also called 'correlation' or 'matching') provides the basis for data fusion. This is the process of linking SAR ship detections and AIS observations considered to be of a common origin. The data association is particularly important for applications and research which use AIS data as a source of ground truth, to both identify and validate ship detections in SAR imagery. This is demonstrated in various 
surveillance applications, including maritime monitoring [2-5], piracy activity [6,7], fishing vessel activity [8-11], polluter identification [12-14] and ice navigation [15]. The detection of non-cooperative (or so-called "dark") ships which have missing AIS information is of particular interest in these applications. This is because ships can be 'dark' for a number of reasons. For example, some ships are exempt from transmitting AIS due to their size and class [1]. Conversely, some ships intentionally avoid detection for both illicit and non-illicit reasons such as carrying out illegal fishing [11] or hiding fishing activities from competitors. There are also a wide range of research activities which use SAR and AIS. Two of the most common research areas include the development and validation of SAR ship detection algorithms [16-21] and the evaluation of SAR ship classification models [22-27]. This also includes the development of open benchmark datasets intended for these research areas [28-30]. Therefore, a robust and accurate method of data association is essential for the rapidly growing number of applications and research involving SAR and AIS.

In the scientific literature, the data association between SAR and AIS has been predominately carried out using simple methods such as Nearest Neighbour (NN) [20,31-35]. The $\mathrm{NN}$ is a form of proximity search that assigns data based on a minimum distance, where the 'distance' is defined by a selected distance metric or threshold. Optimal methods have been implemented such as Global Nearest Neighbour (GNN) [21,36,37], which requires solving the assignment problem $[38,39]$. This involves computing the lowest 'cost' (i.e., the distance) for all assignments and, unlike the suboptimal NN, determines a unique pairing between observations. Additionally, techniques commonly found in pattern recognition and computer vision have been recommended for SAR and AIS data association based on simulated data [40,41], while being implemented for optical and AIS [42]. The basic principle involves recovering the spatial transformation that aligns the positions of the two sets of observations (or point sets). In a previous publication, it is demonstrated that the performance of all of these techniques depends primarily on the temporal difference (or 'gap') between the sensor acquisitions as well as the ship density [43]. In congested areas, ships detected in SAR imagery can be wrongly associated with AIS observations resulting in mis-associations. It is shown that GNN outperforms both $\mathrm{NN}$ and techniques that are based exclusively on spatial transformations. Therefore, a modified GNN approach is implemented in this work.

Two other processes are carried out before data association. The first addresses the temporal difference between the two sensor acquisitions and involves interpolating and/or extrapolating the AIS-reported positions to the time of the SAR image acquisition (also called 'AIS position projection'). In the literature, methods implemented vary from simple linear methods such as dead reckoning $[3,15,33,35,40,44]$ to more involved methods such as spline interpolation [32,34] and knowledge-based interpolation [36]. However, these methods have an upper limit to their performance, where for large temporal differences the uncertainty in the estimated position becomes very large. A technological solution has emerged in new generation spaceborne SAR missions which have the SAR sensor and AIS receiver co-located on the same platform (e.g., NovaSAR-1 [45], PAZ mission [46] and RCM [47]). This allows for nearly contemporaneous data acquisition and alleviates the dependency on interpolation and/or extrapolation methods for large temporal differences. The current trend of growing Sat-AIS and SAR microsatellite constellations also provides a greater chance for data alignment.

The second process deals with the well-known effect observed in SAR imagery that, when an object is moving with a non-zero range velocity component, it will appear displaced in the azimuth direction $[48,49]$. This is known as azimuth image shift (or Doppler shift) and can give rise to the interesting phenomenon where moving ships appear displaced from their wake and wave pattern. The formulae for quantifying this displacement (and equally the radial velocity) for satellite SAR imagery are also well-known [2,50,51]. In studies using SAR and AIS data, the displacement is typically compensated for by using the AIS-reported velocity in conjunction with the satellite and SAR viewing geometry parameters $[31,36,44,52]$. 
The purpose of this study is to demonstrate an improved method that achieves a high level of correspondence between the data, especially in dense shipping environments. The method utilises a SAR ship classification model based on AIS transfer learning, which has recently shown to be an effective alternative to conventional SAR ship classification [53,54]. The returned class information is subsequently used to maximise the confidence in the data association. The data association between SAR and AIS is formulated as an $m$-best twodimensional (2D) assignment problem $[39,55]$, which is solved using the Jonker-Volgenant algorithm [56]. This returns not only the best (optimal) AIS-SAR assignments, but also the second, third, and, in general, the $m$ th best assignments. The advantage of this rankordered approach means that a robust match between the data can be achieved, where class and static information (i.e., the ship type and dimensions) is used to reduce the ambiguity in the association. Two case studies in the UK are used to evaluate the performance of the classification-aided data association technique based on the types of SAR product used for maritime surveillance: wide-area and large-scale data association in the English Channel (i.e., larger swath, lower resolution SAR) and focused data association in the Solent (i.e., smaller swath, higher resolution SAR).

\section{Materials and Methods}

Figure 1 shows a generalised workflow for the method. The fusion workflow is designed to accept input data from various sources as well as SAR imagery products with different imaging modes. The main processes in the workflow include SAR image and AIS data processing, the training of a ship classification model, and data association of the SAR and AIS datasets. The objective of SAR image processing (or ship detection) is to detect objects on the sea surface in the SAR image. The objective of AIS data processing is to prepare AIS data to be matched with the SAR ship detections. The ship classification model uses a transfer learning method to make predictions of the SAR ship detections' ship type. This information is used in the data association which returns a robust match between the SAR $\left(S A R_{D}\right)$ and AIS $\left(A I S_{D}\right)$ datasets. These processes are described in more detail in the following subsections.

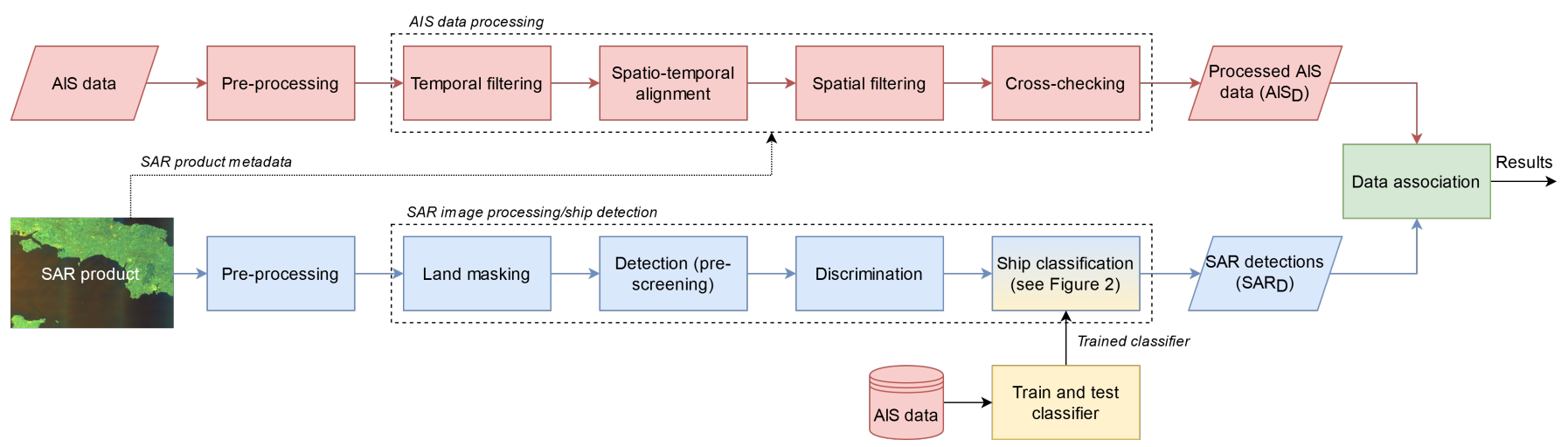

Figure 1. Fusion workflow for SAR and AIS datasets.

\subsection{Ship Detection}

The Search for Unidentified Maritime Objects (SUMO) software is used for ship detection, which is an open-source ship detection software developed by the European Commission's Joint Research Centre (JRC) [57]. The SUMO software implements the SUMO algorithm which is based on a Constant False Alarm Rate (CFAR) detector and uses a K-distribution to model the sea clutter [19]. The SUMO processing parameters are given in Table 1. A $250 \mathrm{~m}$ seaward-buffered land mask is applied to ensure detection is limited to the sea surface and not over land which can give rise to false alarms. The detection threshold values are slightly raised above the default values (by 0.3 as suggested in [57]) in 
order to limit the number of false alarms. However, this also means very small targets may not be detected by the algorithm.

Discrimination involves removing false alarms. The likelihood of a detection being a false alarm is determined by considering its SUMO reliability figure in conjunction with manually inspecting the SAR image. Specifically, the SUMO reliability figure indicates if the object is likely a false alarm or not and, in the case that it is, further indicates if it is an azimuth ambiguity. This information is used when manually inspecting the detection's signature in the SAR image (in context with its surroundings) to help identify false alarms. Additionally, the detection's signature is compared with repeat-pass optical satellite imagery to determine if it is a fixed structure such as a sea fort. If a detection is determined to be a false alarm, it is categorised into what is believed to be its cause or source.

Table 1. SUMO: SAR processing parameters.

\begin{tabular}{ll}
\hline Parameter & Description \\
\hline Algorithm (detector) & CFAR (K-distribution) \\
\hline Nominal false alarm rate & $10^{-7}$ \\
\hline Land mask & OpenStreetMap (250 m buffer) \\
\hline Detection threshold adjustment & $1.8(\mathrm{VV}) 1.5(\mathrm{VH})$ \\
\hline
\end{tabular}

\subsection{AIS Data Processing}

Figure 1 shows the main AIS data processing steps which are carried out sequentially. A step-by-step procedure is given as follows:

1. Temporal filtering:

(a) The AIS dataset is first filtered to the acquisition date of the SAR image and then further filtered to a time interval, $X$, centred on the sensing start time of the SAR image, $T_{S A R}$. This defines a time range of $T_{S A R} \pm \frac{X}{2}$. Ideally, a time interval is selected which allows for the interpolation between at least two positions (for a given ship). However, this is non-trivial as ships often do not comply with the technical standard [58]. This means selecting a time interval based on the maximum reporting interval of AIS is unsuitable. Instead, the time interval is empirically determined based on the average reporting interval for the area of interest.

2. Spatio-temporal alignment:

(a) A cubic Hermite spline interpolation is applied to the track of each ship to determine its position (in latitude and in longitude), Speed Over Ground (SOG) and Course Over Ground (COG) at $T_{S A R}$. A track is the history of a ship's location and is generated by aggregating positions with the same unique Maritime Mobile Service Identity (MMSI) number. If a track cannot be generated (i.e., only a single position is available), then no interpolation is carried out.

(b) Azimuth image shift compensation is carried out on the AIS data. The azimuth shift, $\Delta_{a z}$ in metres, for a moving ship is given by

$$
\Delta_{a z}=\frac{H \widetilde{S O G} \tan \theta \cos \phi}{V},
$$

where $H$ is the satellite (or SAR) altitude in metres, $\widetilde{S O G}$ is the interpolated SOG of the ship in metres per second, $\theta$ is the SAR incidence angle extracted at the AIS-reported position in degrees, $\phi$ is the interpolated COG of the ship relative to the SAR range direction in degrees and $V$ is the magnitude of the 
satellite velocity in metres per second. The AIS-reported position of the ship is then reckoned by the calculated azimuth shift in its opposite relative azimuth direction.

3. Spatial filtering:

(a) The dataset is filtered again according to the spatial extent (or footprint) of the SAR image.

(b) AIS data located within the SAR land mask (including the $250 \mathrm{~m}$ buffer) are also removed.

4. Cross-checking:

(a) The AIS dataset is cross-checked against an open ship database (e.g., ShipAIS [59]) to verify the accuracy of the static data (i.e., length, width and ship type). Missing or invalid entries are also updated using the International Maritime Organization (IMO) number to form a more complete dataset for data association. (Note that, if the IMO number is not available, then the MMSI number is used instead.)

It is important to note that interpolation should be carried out before spatially filtering the AIS dataset to the footprint of the SAR image; otherwise, some AIS data may be missed or mistakenly included. This is because AIS data initially located outside the footprint can be subsequently located inside the footprint after interpolation (and vice versa). Equation (1) is a modified version of Equation (1) in [50], which is implemented as an algorithm. Instead of using SAR information, the algorithm is based on information either directly reported by AIS (i.e., SOG and COG) or by what can be derived such as the SAR incidence angle at the AIS-reported position (instead of the SAR detection position). Consequently, the azimuth shift process is fast and automatic, which is ideal for the situation where there are a large number of ships in the scene. Following the above procedure, the AIS dataset, $A I S_{D}$, is ready for data association.

\subsection{Ship Classification}

The ship classification model makes predictions on the type of ship in SAR imagery. Specifically, the model is first trained (and tested) on AIS data and then transferred to make predictions on SAR ship detections. This method is known as transfer learning and has shown to be effective for SAR ship classification. This is due to the extensive volume and availability of labelled AIS data, making it ideal for machine learning. The objective is to use class information (i.e., ship type) to maximise the confidence in the association between $S A R_{D}$ and $A I S_{D}$. Figure 2 shows a generalised workflow for generating the ship classification model.



Figure 2. Ship classification model workflow.

A step-by-step procedure is given as follows:

1. Import data: The training data $\left(A I S_{C}\right)$ are imported from an AIS database. The database is typically formed from historical/archive AIS data.

2. Preprocess data: The training data are preprocessed by removing anomalous, missing, invalid and duplicated entries that may negatively affect the training of the model.

3. Feature selection: Relevant features (or predictors) for use in training the model are selected (see Table 2). Importantly, since a transfer learning method is used, the selected features from the AIS data are limited to what can also be extracted and/or derived from the SAR ship detections.

4. Feature engineering: New features are derived to improve the predictive power of the model. 
5. Train model(s): Multiple classification algorithms are iteratively trained and tested based on the selected and derived features in order to find the best model that predicts the type of ship.

6. Export model: The best trained model is exported to make ship type predictions on new data (i.e., SAR ship detections, $S A R_{D}$ ). These predictions are subsequently used in the data association.

The specific parameters used to generate the ship classification model are given in Table 2. The classification model is implemented in the MATLAB ${ }^{\circledR}$ environment. The ship classification model considers only unique ships based on the MMSI number. This is because only features based on static data (i.e., length, width and aspect ratio) are used in training the model and so duplicate entries of the same ship can introduce bias. This results in a relatively simple but powerful classification model. Additionally, geolocation data (i.e., latitude and longitude) can be used directly in training a model [54] or by first clustering the data to reduce its dimensionality [60].

The ship classification model implements the Random Undersampling Boosting (RUSBoost) algorithm [61]. There are two reasons for selecting this algorithm. Firstly, it is particularly effective at classifying imbalanced data where some classes in the training data have significantly fewer observations than other classes. This is commonly exhibited in AIS data and the distribution or skewness of the classes (ship types) will generally vary according to the geographical area as well as the time in the shipping season. Secondly, the RUSBoost algorithm consistently outperforms other ensemble algorithms that do not implement data sampling techniques (in terms of the true positive rate and false negative rate for each class), and is computationally less expensive than other hybrid data sampling/boosting algorithms such as SMOTEBoost [62].

Table 2. Ship classification model parameters.

\begin{tabular}{ll}
\hline Parameter & Description \\
\hline $\begin{array}{l}\text { Feature selection } \\
\text { (input features) }\end{array}$ & $\begin{array}{l}\text { Ship length }(l) \\
\text { Ship width }(w)\end{array}$ \\
\hline $\begin{array}{l}\text { Feature engineering } \\
\text { (derived features) }\end{array}$ & $\begin{array}{l}\text { Length-to-width aspect ratio }(l / w) \\
\text { Width-to-length aspect ratio }(w / l)\end{array}$ \\
\hline & Ship type (six classes): \\
& - Cargo \\
Response & - Passenger \\
& - Pleasure \\
& - Tanker \\
& Tug \\
\hline Algorithm & RUSBoost [61] \\
\hline Model assessment method & k-fold cross-validation $(\mathrm{k}=10)$ \\
\hline
\end{tabular}

The AIS training data, $A I S_{C}$, totals 20,177 unique entries. Figure 3 shows the number of unique ships for each response class in the AIS training data. The 'Other' and 'NA (Not Available)' ship types as well as those with fewer than one percent of the total number of observations (e.g., 'Military') are excluded from the AIS training data (shown in grey).

Figure $4 a, b$ show the length and width distributions in the AIS training data for each ship type, respectively. There is a clear distinction between the dimensions of the group fishing, pleasure and tug ship types and the pair cargo and tanker ship types. In contrast, the dimensions of the passenger ship type are more uniformly distributed. In order to find a further distinction between these sub-groups (i.e., discern between a cargo and tanker), new features or predictors are derived (i.e., feature engineering). Figure $5 a, b$ 
show the width-to-length and length-to-width aspect ratio distributions for each ship type, respectively.

It is evident from Figure 3 that there is a class imbalance in the training data. In other words, there is a significant disparity between the number of observations in each class (or ship type). For example, the pleasure ship type has nearly 11 times the number of observations compared to the tug ship type. The classification model uses 10-fold crossvalidation to prevent overfitting during training. The trained model results and MATLAB ${ }^{\circledR}$ model parameters are given in Table 3.



Figure 3. Training data $\left(A I S_{C}\right)$ ship type distribution.



(a)

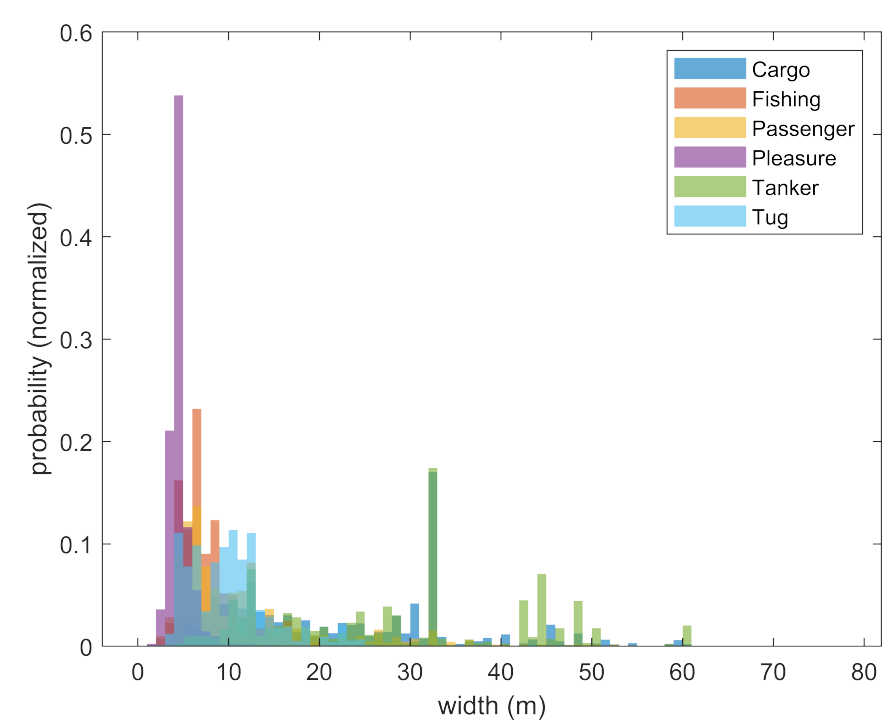

(b)

Figure 4. Training data $\left(A I S_{C}\right)$ (a) length distribution (grouped into bins of $5 \mathrm{~m}$ ) and (b) width distribution (grouped into bins of $1 \mathrm{~m}$ ) for each ship type. 




(a)

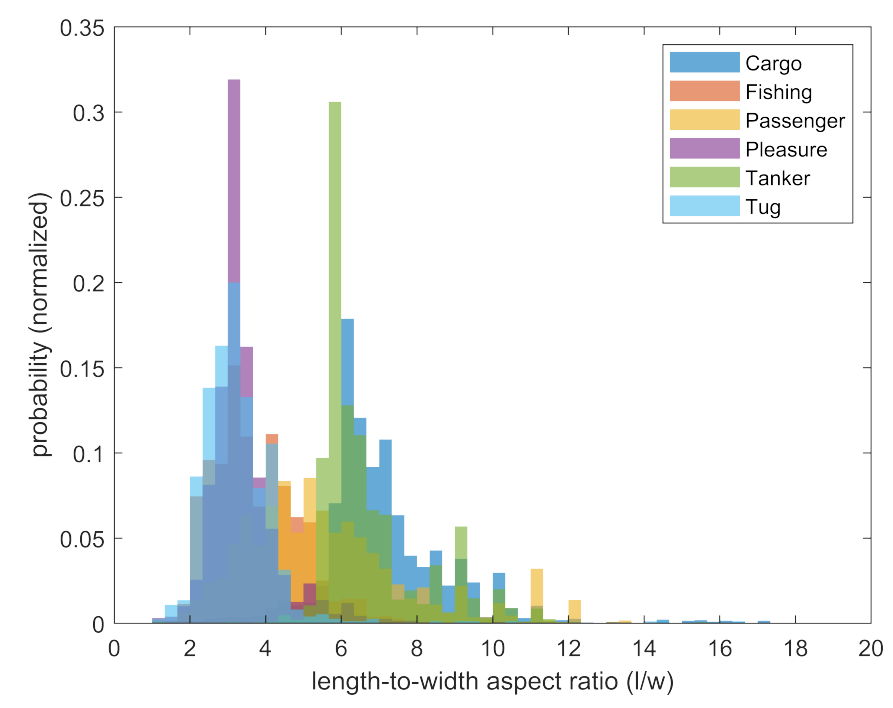

(b)

Figure 5. Training data $\left(A I S_{C}\right)(\mathbf{a})$ width-to-length aspect ratio $(w / l)$ distribution and $(\mathbf{b})$ length-to-width aspect ratio $(l / w)$ distribution for each ship type.

Table 3. Trained model results and MATLAB ${ }^{\circledR}$ model parameters.

\begin{tabular}{ll}
\hline \multicolumn{1}{c}{ Model results } & \multicolumn{1}{c}{ Value } \\
\hline Accuracy & $68.6 \%$ \\
\hline Total misclassification cost & 6340 \\
\hline Prediction speed (approx.) & $41,000 \mathrm{obs} / \mathrm{s}$ \\
\hline Training time * & $16.367 \mathrm{~s}$ \\
\hline \multicolumn{1}{c}{ Model parameters } & RUSBoosted Trees \\
\hline Preset & RUSBoost \\
\hline Ensemble method & Decision tree \\
\hline Learner type & 20 \\
\hline Max. number of splits & 50 \\
\hline Number of learners & 0.1 \\
\hline Learning rate & $10-$ fold cross-validation \\
\hline Validation &
\end{tabular}

\subsection{Data Association}

The data association of SAR ship detections and AIS data are twofold. Firstly, an $m$-best assignment technique is implemented to assign AIS data $\left(A I S_{D}\right)$ to SAR ship detections $\left(S A R_{D}\right)$. Importantly, this technique ranks all the assignments in the order of increasing cost. The cost is defined as the distance for each AIS-SAR pair, which is computed using the geodesic distance. The assignments are made by minimising the sum of the total distances for all possible AIS-SAR pairings. Specifically, the Jonker-Volgenant algorithm [56] is used to find an optimal solution to the GNN assignment problem. Moreover, the value selected for $m$ is three meanings for which, for a given SAR ship detection, the top 3-best assignments in $A I S_{D}$ are returned. The performance of this particular technique has been quantitatively assessed in a previous publication [43].

Secondly, once rank-ordered assignments are made, confidence levels are determined according to the criteria specified in Table 4. Specifically, the assignments' geometric (i.e., 
length and width) and class (i.e., ship type) data are compared, which are considered to be the most reliable static data fields. The assignments' geometric data are in agreement if their absolute difference is within a specified threshold, $t$. Similarly, the class data are in agreement if the SAR ship classification matches the one reported by AIS. After the comparison, the confidence level is determined based on the number of geometric and class data in agreement. The assignment with the highest confidence level (out of the total three) is selected as the final assignment. Competing assignments with the same confidence level are resolved by selecting the highest ranking pair.

Figure 6 shows a conceptual example of how the final assignment is determined. The top 3-best AIS assignments are returned for a single SAR ship detection. The candidate assignments are ordered in terms of their rank. The AIS candidates' reported length, width and ship type are compared with those of the SAR ship detection. (Recall that the SAR ship detection's geometric and class data are determined in Sections 2.1 and 2.3, respectively.) A confidence level is given to each of the three AIS candidates according to the criteria specified in Table 4. In the example, the final assignment is $\mathrm{A}_{1}$. Note that the highest ranked assignment, $A_{2}$, is superseded by the third highest ranked assignment, $A_{1}$. The algorithm correctly updates the final assignment to $A_{1}$ which has better agreement with the SAR ship detection.

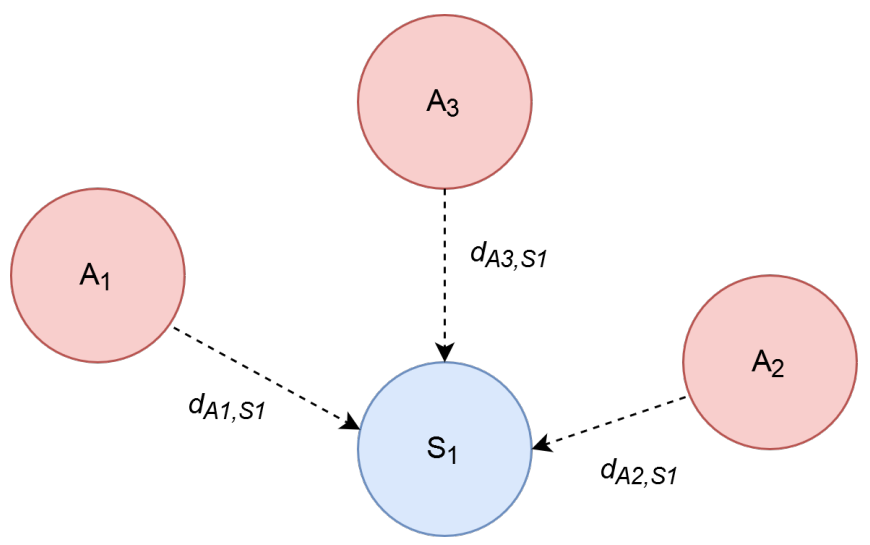

\begin{tabular}{|c|c|c|c|}
\hline SAR $_{\mathbf{D}}$ & Rank & AIS $_{\mathbf{D}}$ & Confidence \\
\hline \multirow{3}{*}{$\mathrm{S}_{1}$} & 1 & $\mathrm{~A}_{2}$ & Low \\
\cline { 2 - 4 } & 2 & $\mathrm{~A}_{3}$ & Low \\
\cline { 2 - 4 } & 3 & $\mathrm{~A}_{1}$ & High \\
\hline
\end{tabular}

Figure 6. Final assignment concept.

Table 4. Determination of confidence level.

\begin{tabular}{|c|c|c|c|}
\hline Confidence & $\begin{array}{c}\text { Length, } l \\
\left(S A R_{D_{l}}-A I S_{D_{l}} \leq t_{l}\right)\end{array}$ & $\begin{array}{c}\text { Width, } w \\
\left(S A R_{D_{w}}-A I S_{D_{w}} \leq t_{w}\right)\end{array}$ & $\begin{array}{c}\text { Ship Type } \\
\left(S A R_{D_{\text {ShipType }}} \equiv A I S_{D_{\text {ShipType }}}\right)\end{array}$ \\
\hline Low & $\mathrm{F}$ & F & $\mathrm{F}$ \\
\hline \multirow{3}{*}{ Medium } & $\mathrm{T}$ & $F$ & $\mathrm{~F}$ \\
\hline & $\mathrm{F}$ & $\mathrm{T}$ & $\mathrm{F}$ \\
\hline & $\bar{F}$ & $F$ & $\mathrm{~T}$ \\
\hline \multirow{3}{*}{ High } & $\mathrm{T}$ & $\mathrm{T}$ & $\mathrm{F}$ \\
\hline & $\mathrm{T}$ & $\mathrm{F}$ & $\mathrm{T}$ \\
\hline & $F$ & $\mathrm{~T}$ & $\mathrm{~T}$ \\
\hline Very High & $\mathrm{T}$ & $\mathrm{T}$ & $\mathrm{T}$ \\
\hline
\end{tabular}

(T: True; F: False).

\section{Results}

3.1. Case Study A: English Channel, UK

\subsubsection{Product Details}

The Sentinel-1 SAR product is acquired in the English Channel, UK. A preview of the SAR product is shown in Figure 7 with details given in Table 5. The Interferometric Wide (IW) swath mode of Sentinel-1 is particularly suited for wide-area maritime surveillance offering a $250 \mathrm{~km}$ swath at $20 \mathrm{~m}$ by $22 \mathrm{~m}$ spatial resolution (multilooked). Figure 8 shows 
the coverage area of the AIS data used for data association $\left(A I S_{D}\right)$. The data provided by LuxSpace Sàrl cover the time period 1 October 2017 to 30 November 2017 (two months), and comprises terrestrial and satellite-AIS (Sat-AIS) data. The shipping density in the coverage area is particularly very high with parts showing on average between 10,000 and 100,000 annual vessel transits. The AIS temporal coverage around $T_{S A R}$ (24 October 2017) for the area shown in Figure 8 is also checked to ensure that there is continuous data reception with no data gaps.



Figure 7. English Channel SAR product (VV polarisation).

Table 5. English Channel SAR product details.

\begin{tabular}{ll}
\hline Parameter & Description \\
\hline Datetime (UTC) & 2017-10-24T06:23:21.314Z \\
\hline Instrument & SAR-C \\
\hline Mode & IW \\
\hline Satellite & Sentinel-1A \\
\hline Spatial resolution & $20 \times 22$ m $($ range $\times$ azimuth) \\
\hline Pass direction & Descending \\
\hline Polarisation & VV VH \\
\hline Product level & Level-1 \\
\hline Product type & GRD \\
\hline Product identifier & S1A_IW_GRDH_1SDV_20171024T062321_20171024T062346_ \\
\hline
\end{tabular}






Figure 8. English Channel AIS coverage area (2 km grid resolution).

\subsubsection{Ship Detection}

Ship detection follows Section 2.1. Information on the processing parameters is given in Table 1. In total, SUMO returns 286 detections or targets in the SAR image. After discrimination, 102 detections are considered to be false alarms. These are subsequently removed resulting in a new total of 184 detections. The majority of false alarms (58.8\%) arise from an imperfect land mask (see Figure 9).

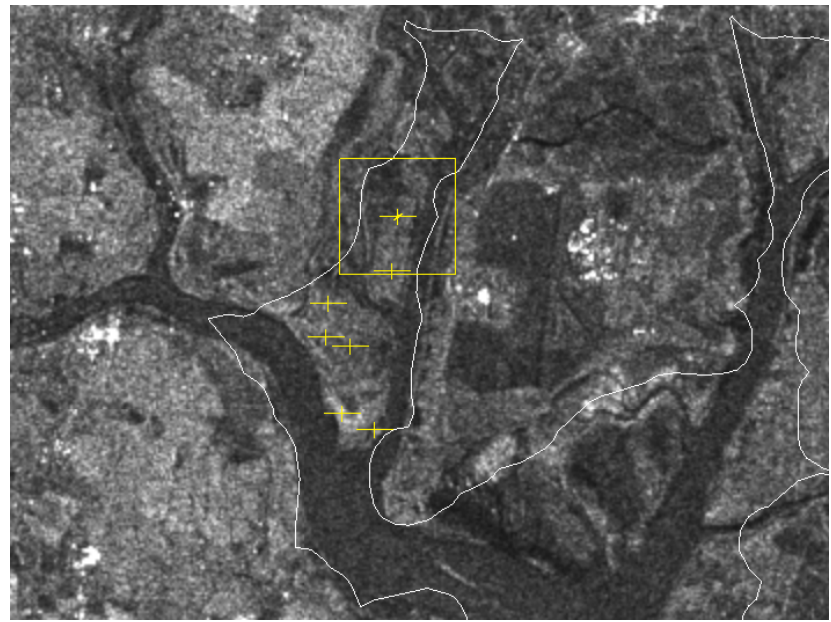

(a)

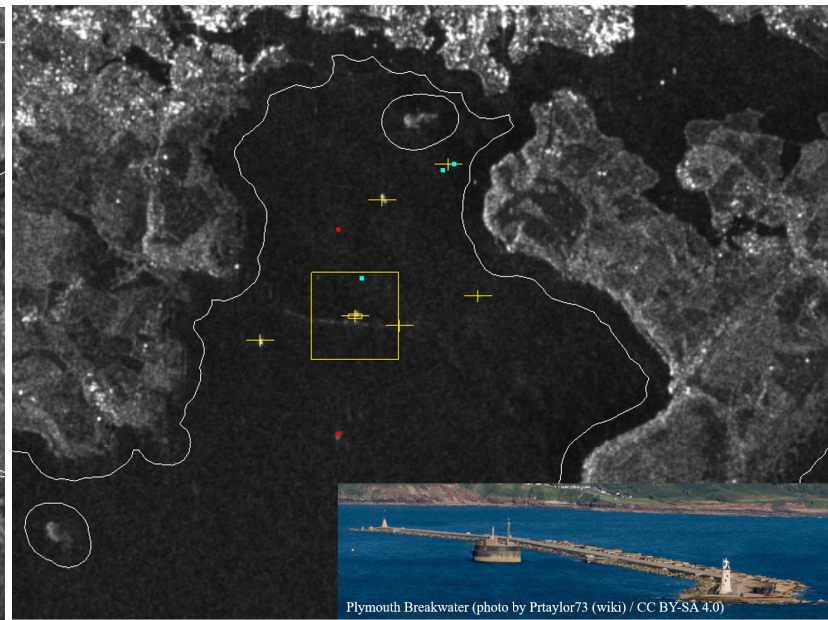

(b)

Figure 9. English Channel SUMO false alarm examples: (a) imperfect land mask; (b) coastal infrastructure (yellow box) and azimuth ambiguities (red points). 


\subsubsection{AIS Data Processing}

The AIS data to be associated with the detections in the SAR image $\left(A I S_{D}\right)$ follow the processes described in Section 2.2. For temporal filtering, a time interval, $X$, of $40 \mathrm{~min}$ is selected meaning AIS data within 20 min either side of the sensing start time of the SAR image, $T_{S A R}$, are considered for association. This value is determined by measuring the percentage of AIS data that are successfully interpolated to $T_{S A R}$ as a function of $X$ (see Figure 10). A suitable time interval is one with a high percentage of successful interpolations for the majority of data whilst keeping the number of unique ships constant (i.e., limiting the number of ships with a single position in the interpolation as $X$ increases). Figure 10 shows that, for the time interval $X=40 \mathrm{~min}$, approximately $95 \%$ of the AIS data are successfully interpolated to $T_{S A R}(06: 23: 21 \mathrm{UTC})$, and little performance is gained beyond $X=40 \mathrm{~min}$. Figure 11 also shows the average elapsed time between consecutive timestamps of the same ship, for all ships during October 2017 in the English Channel. The majority of ships have an average reporting interval in the range of 5-30 min further supporting a time interval selection of $X=40 \mathrm{~min}$ (i.e., $T_{S A R} \pm 20 \mathrm{~min}$ ). Figure 12 shows three visualisations of the interpolation results.

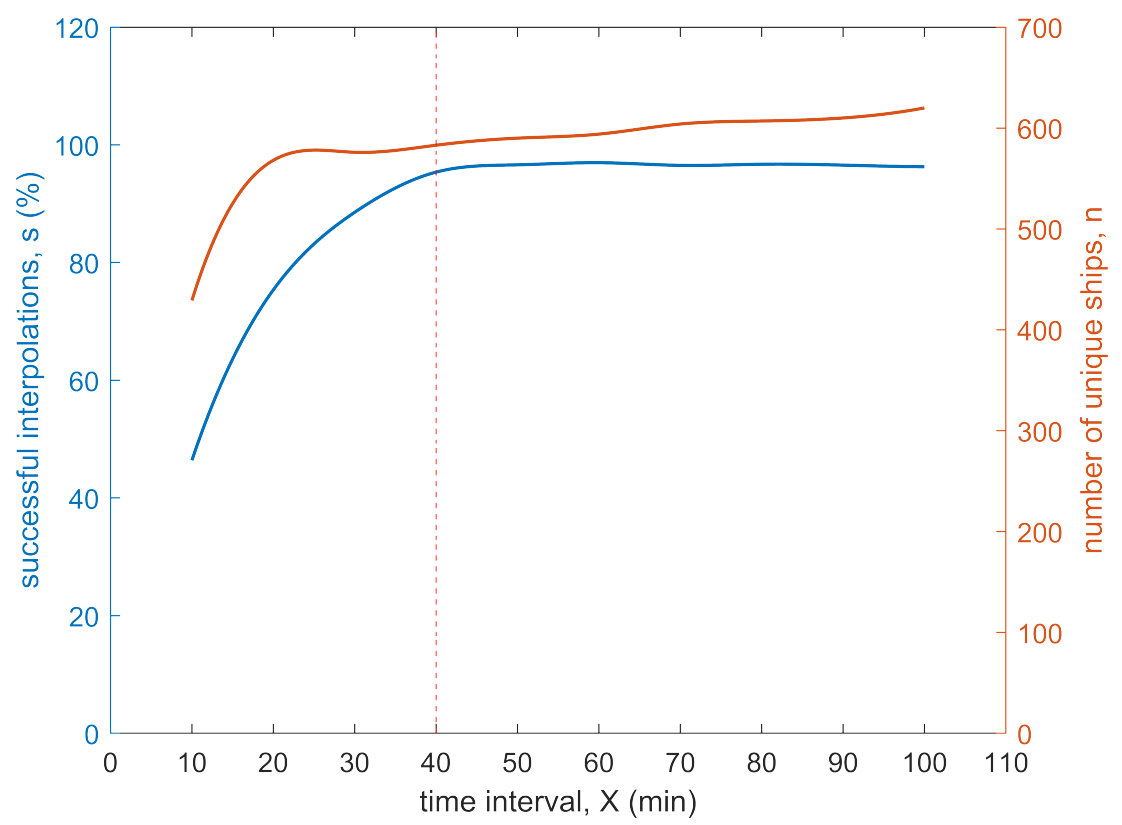

Figure 10. English Channel time interval selection $(X=40 \mathrm{~min})$.

Following interpolation and spatial filtering to the SAR image footprint, AIS data located within the SAR land mask (including the $250 \mathrm{~m}$ buffer) are removed. This results in a final count of 199 unique ships, of which nine (4.5\%) are unsuccessfully interpolated to $T_{S A R}$ with an average time deviation of $10 \mathrm{~min} 35 \mathrm{~s}$. Additionally, the average azimuth shift for the final count is $192.5 \mathrm{~m}$, which is based on ships with a valid and non-zero velocity (191 total). The azimuth shift is compensated for using Equation (1).

Finally, the dataset is cross-checked against a database. Table 6 gives the number of missing values in each field both before and after cross-checking the AIS data as well as the percentage change. After cross-checking, the final dataset has less than $10 \%$ of values missing in each static data field. 




Figure 11. English Channel average reporting interval of the same ship (MMSI), for all ships during October 2017. (Note that ships with a single timestamp are omitted (total: 3123).)


Figure 12. English Channel interpolation examples. 
Table 6. English Channel missing data before and after cross-checking AIS data (total: 199).

\begin{tabular}{cccccc}
\hline & \multicolumn{2}{c}{ Before } & \multicolumn{2}{c}{ After } & \multirow{2}{*}{ Change (\%) } \\
\cline { 1 - 5 } Data Field & \# Missing & \% of Total & \# Missing & \% of Total & \\
\hline Length & 38 & 19.2 & 14 & 7.1 & -12.1 \\
\hline Width & 42 & 21.2 & 18 & 9.1 & -12.1 \\
\hline Ship type & 28 & 14.1 & 6 & 3.0 & -11.1 \\
\hline
\end{tabular}

\subsubsection{Classification-Aided Data Association}

The total number of SAR ship detections $\left(S A R_{D}\right)$ and unique AIS data points $\left(A I S_{D}\right)$, as well as their respective number of assignments and unassignments, are given in Table 7. Figure 13 shows a visualisation of these results. The agreement in the assignments' geometric and class data is also given in Table 7. The selected geometric thresholds (see Table 4) are $t_{l}=25 \mathrm{~m}$ (2.5 pixels) and $t_{w}=10 \mathrm{~m}$ (1 pixel) for the length and width, respectively.

Table 7. English Channel total number of (top) assignments and unassignments (bottom) features in agreement.

\begin{tabular}{|c|c|c|}
\hline & $S A R_{D}$ & $A I S_{D}$ \\
\hline Total & 184 & 199 \\
\hline Assigned & \multicolumn{2}{|c|}{152} \\
\hline$\%$ of Total & 82.6 & 76.4 \\
\hline Unassigned & 32 & 47 \\
\hline$\%$ of Total & 17.4 & 23.6 \\
\hline Assigned & Feature & \# of matches \\
\hline \multirow{3}{*}{152} & Length (valid: 146) & $55(37.7 \%)$ \\
\hline & Width (valid: 141) & $129(91.5 \%)$ \\
\hline & Ship type (valid: 135 ) & $34(25.2 \%)$ \\
\hline
\end{tabular}

Figure 14a shows the estimated SAR length returned by the SUMO algorithm plotted against the reported AIS length for each assignment. The SAR-estimated lengths show moderate agreement with AIS-reported lengths greater than $100 \mathrm{~m}$. Conversely, SAR-estimated lengths are considerably overestimated for AIS-reported lengths below $100 \mathrm{~m}$, especially at $20 \mathrm{~m}$ (approximately). Figure 14b shows a similar plot for widths. The SAR-estimated widths show a consistent overestimation for all AIS-reported widths. The overestimation by the SUMO algorithm is corrected for by establishing the relationship between the estimated SAR width and AIS-reported width (of the form $y=m x+c$ ). This is done using linear regression; specifically, a robust least-squares fit with bisquare weights is used to estimate the parameters (i.e., gradient, $m$ and intercept, $c$ ) of the relationship. The inverse of this relationship is used to update the estimated SAR width values. Figure 14c shows the updated values of the estimated SAR width plotted against the reported AIS width for each assignment. In comparison to Figure 14b, the gradient is now very close to an ideal value of one.

The general overestimation in SAR-estimated dimensions can be explained by the variability in a target's SAR signature [63]. For example, some overestimations are likely a consequence of azimuth blurring, which is a form of motion-induced distortion sometimes found in SAR imagery [48]. This is caused by the motion of a target such as a ship relative to the radar (i.e., moving linearly in the azimuth direction). (This is a similar effect to azimuth shift where moving targets in the range direction appear displaced in azimuth.) Reportedly, this blurring can be of the order of $100 \mathrm{~m}$ giving rise to significant overestimations of the ship's dimensions, especially for small ships $[19,64]$. 




Figure 13. English Channel data association.

A histogram of the confidence levels of the 152 assignments is shown in Figure 15a. In total, 34 assignments (25.2\%) (out of a maximum of 135 AIS data points with a valid ship type) received an improved confidence level due to a match in their ship type. These assignments would have otherwise had a lower confidence level if ship classification were not implemented.

In total, 47 AIS data points (23.6\%) and 32 SAR detections (17.4\%) are unassigned. After visually inspecting the SAR signatures of the unassigned AIS data points in the SAR image, it is observed that:

- $\quad$ Four (4) are due to a discrepancy between the SAR footprint and the SAR image. The main reason is the SAR image contains noise (or artefacts) at its borders (visible in Figure 7). (This noise is common to Sentinel-1 Level-1 GRD products after being processed from RAW data.) The extent of the SAR footprint includes these areas of border noise where AIS data points may be located but no SAR detections.

- $\quad$ Seven (7) are unsuccessfully interpolated to $T_{S A R}$, meaning their true positions have a greater associated uncertainty and are less likely to be assigned.

- $\quad 11$ are moored to structures such as piers and oil terminals located within ports and harbours (located outside the land mask). These structures merge with or deform the SAR signature in such a way that leads to no SAR detection.

- 20 are either below the selected SUMO detection threshold or have a very weak SAR signature that is below the limit of detectability of the SAR sensor. These are generally small ships. For example, the average ship length of the 20 unassigned AIS data points is $16.3 \mathrm{~m}$ where most are fishing vessels.

There are 32 unassigned SAR detections. After visually inspecting the unassigned SAR detections in the SAR image, it is observed that 14 are false alarms which have been missed 
by initial inspection (see Section 3.1.2). The remaining 18 unassigned SAR detections are thought to be 'dark' ships.

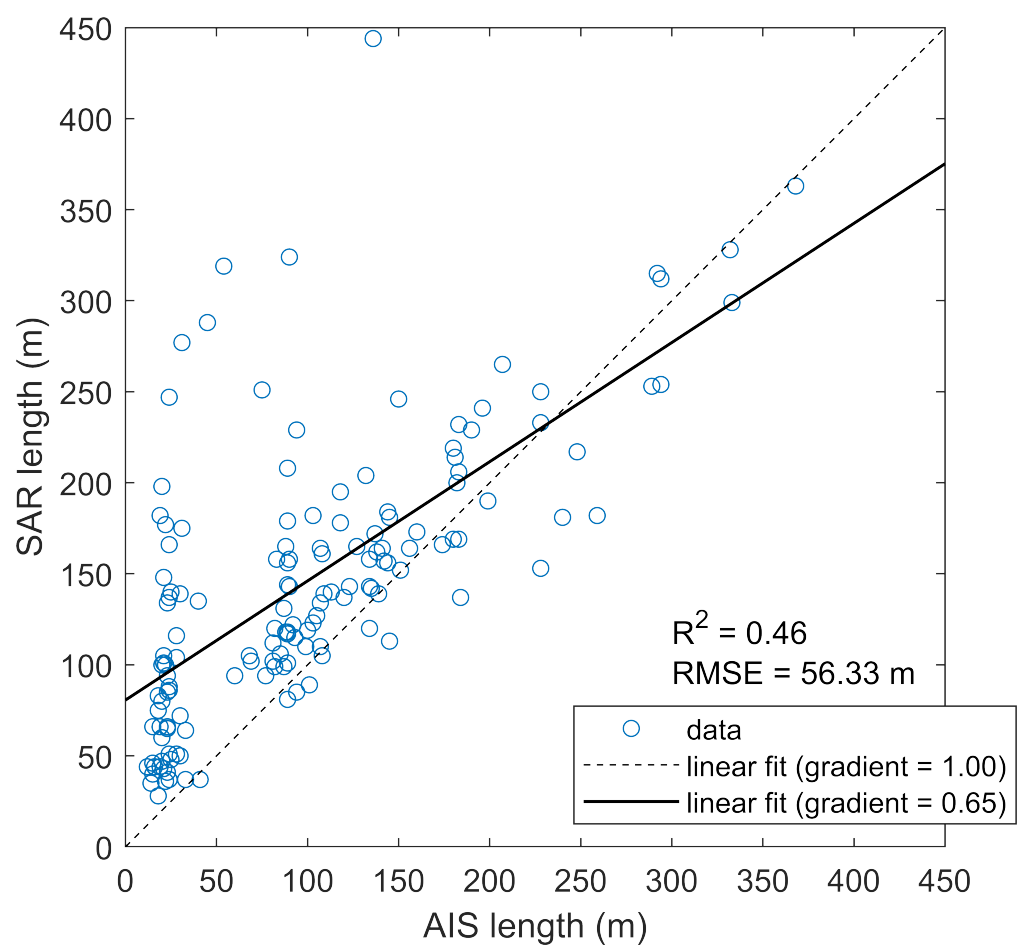

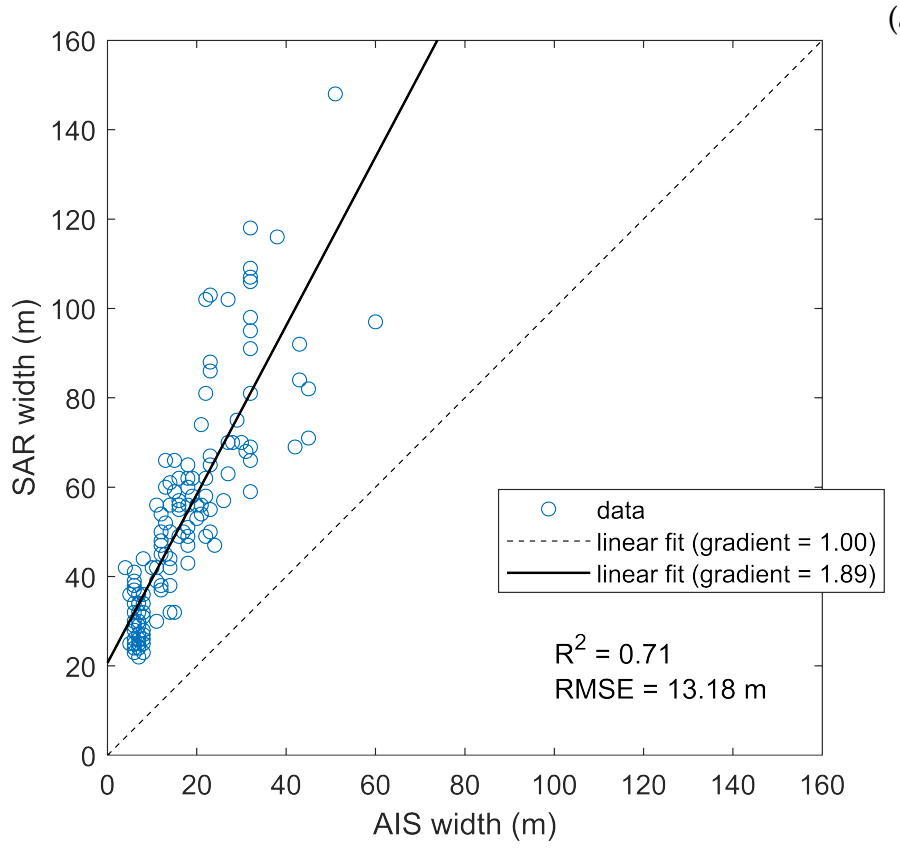

(b) (a)



(c)

Figure 14. English Channel estimated SAR (a) length; (b) width; (c) updated width; against reported AIS for each assignment.

Figure $15 \mathrm{~b}$ shows the distribution of AIS-SAR assignment (or matching) distances. The average distance is $160.9 \mathrm{~m}$ with the majority having a distance less than $100 \mathrm{~m}$. This discrepancy can be explained by small errors in the interpolation and azimuth shift compensation processes and, to a lesser extent, georeferencing errors introduced from the SAR coordinate transformation. Additionally, both sensors have an associated uncertainty in their geolocation accuracy. The Sentinel-1 Interferometric Wide (IW) swath mode 
typically has a geolocation accuracy of $7 \mathrm{~m}$ [65], while AIS indicates the position accuracy as either 'high' ( $\leq 10 \mathrm{~m})$ or 'low' (>10 m, default) [1].

Three AIS-SAR assignments have a distance greater than one kilometre. One is from removing a SAR detection incorrectly identified as an azimuth ambiguity by the SUMO algorithm. This caused the AIS data point to match with a SAR detection located at a much greater distance. The other two are related to the interpolation process with one being unsuccessfully interpolated to $T_{S A R}$ (time deviation of $18 \mathrm{~min} 28 \mathrm{~s}$ ), and the other having an undulation that overshoots at $T_{S A R}$. The average matching distance is $133.0 \mathrm{~m}$ excluding these three outliers.



(a)

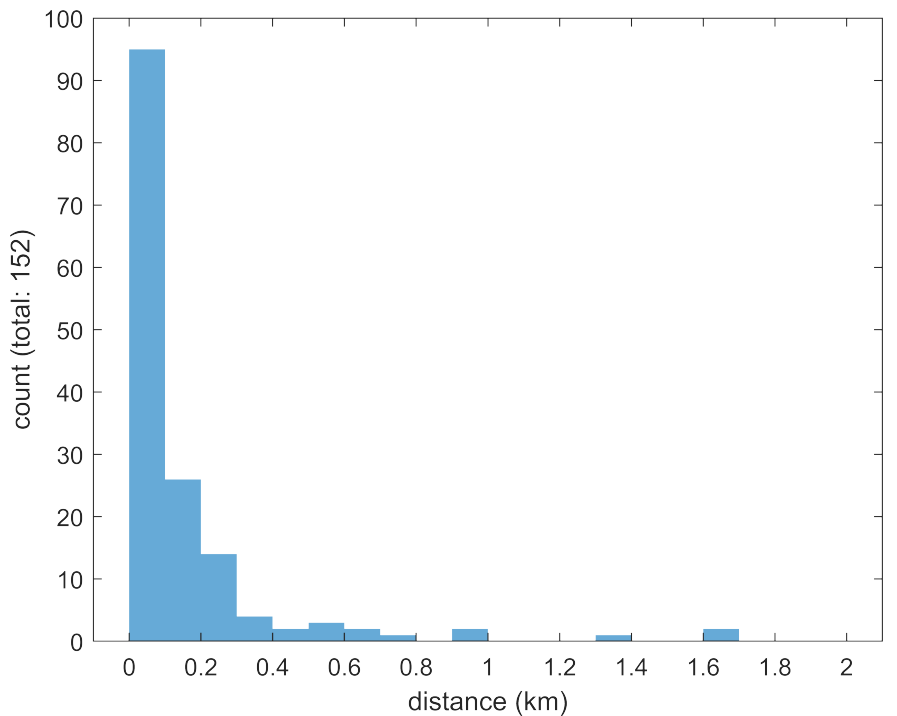

(b)

Figure 15. English Channel (a) AIS-SAR assignment confidence levels and (b) distribution of assignment distances.

\subsection{Case Study B: The Solent, UK}

\subsubsection{Product Details}

The ICEYE-X2 SAR product is acquired in the Solent, UK. A preview of the SAR product is shown in Figure 16 with details given in Table 8. The Stripmap imaging mode is particularly suited for focused maritime surveillance offering a $30 \mathrm{~km}$ swath at $3 \mathrm{~m}$ by $3 \mathrm{~m}$ spatial resolution (multilooked). The coverage area of the AIS data used for data association $\left(A I S_{D}\right)$ is the same as the SAR footprint. The data provided by Spire Maritime covers $24 \mathrm{~h}$ on 30 October 2020 and comprises of terrestrial and satellite-AIS (Sat-AIS) data.

Table 8. The Solent SAR product details.

\begin{tabular}{ll}
\hline Parameter & Description \\
\hline Datetime (UTC) & 2020-10-30T10:43:22.739 \\
\hline Mode & Stripmap \\
\hline Satellite & ICEYE-X2 \\
\hline Spatial resolution & $3 \times 3 \mathrm{~m}($ range $\times$ azimuth) \\
\hline Pass direction & Descending \\
\hline Polarisation & VV \\
\hline Product type & GRD \\
\hline Product identifier & ICEYE_X2_GRD_SM_36769_20201030T104322 \\
\hline
\end{tabular}






Figure 16. The Solent SAR product (VV polarisation).

\subsubsection{Ship Detection}

The SUMO ship detection software does not currently support ICEYE products; therefore, ship detection is carried out in the Sentinel Application Platform (SNAP) which uses a CFAR algorithm. The SNAP processing parameters are given in Table 9.

Table 9. SNAP: SAR processing parameters.

\begin{tabular}{|c|c|}
\hline Parameter & Description \\
\hline Application & SNAP (version 7.0) \\
\hline Calibration & Output sigma0 band \\
\hline Land mask & OpenStreetMap (50 m buffer) \\
\hline Algorithm (detector) & Two-parameter CFAR \\
\hline Adaptive thresholding & $\begin{array}{l}\text { Target Window Size }(\mathrm{m}): 20 \\
\text { Guard Window Size }(\mathrm{m}): 500 \\
\text { Background Window Size (m): } 800 \\
\text { PFA: } 10^{-7}\end{array}$ \\
\hline Object discrimination & $\begin{array}{l}\text { Object dimension threshold: } \\
\text { Min. Target Size (m): } 10 \\
\text { Max. Target Size (m): } 600\end{array}$ \\
\hline
\end{tabular}




\subsubsection{AIS Data Processing}

The AIS data processing follows a similar process to Section 3.1.3. For temporal filtering a time interval, $X$, of $60 \mathrm{~min}$ is selected. Following interpolation and spatial filtering, a final count of 45 unique ships are returned, of which two are unsuccessfully interpolated to $T_{S A R}(10: 43: 22$ UTC).

\subsubsection{Classification-Aided Data Association}

The total number of SAR ship detections $\left(S A R_{D}\right)$ and unique AIS data points $\left(A I S_{D}\right)$, as well as their respective number of assignments and unassignments, are given in Table 10 . Figure 17 shows a visualisation of these results. The agreement in the assignments' geometric and class data are also given in Table 10. The selected geometric thresholds (see Table 4) are $t_{l}=12.5 \mathrm{~m}$ (5 pixels) and $t_{w}=7.5 \mathrm{~m}$ ( 3 pixels) for the length and width, respectively.

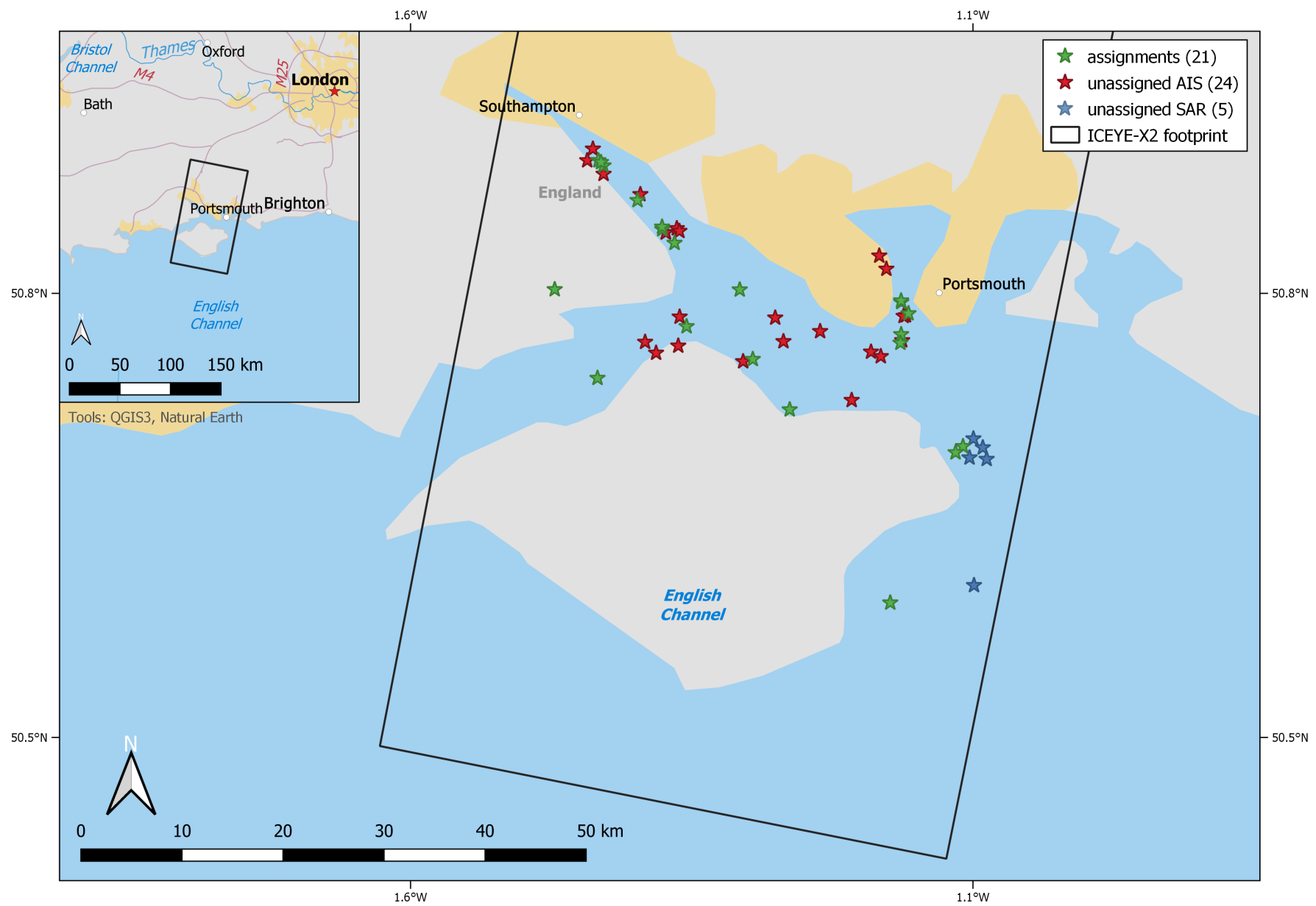

Figure 17. The Solent data association.

Figure 18a shows the estimated SAR length plotted against the reported AIS length for each assignment. The SAR-estimated lengths show good agreement with AIS-reported lengths. Figure $18 \mathrm{~b}$ shows a similar plot for widths. Similarly, the SAR-estimated widths show a good agreement with AIS-reported widths.

A histogram of the confidence levels of the 21 assignments is shown in Figure 19a. The majority of assignments received a 'Very High' confidence level. In total, 13 assignments (76.5\%) (out of a maximum of 17 AIS data points with a valid ship type) received an improved confidence level due to a match in their ship type. These assignments would have otherwise had a lower confidence level if ship classification were not implemented.

In total, 24 AIS data points (53.3\%) and five SAR detections (19.2\%) are unassigned. After visually inspecting the SAR signatures of the unassigned AIS data points in the 
SAR image, it is observed that: three (3) are moored to structures (such as a tug pontoon) that deform the SAR signature in such a way that leads to no SAR detection; and 21 are small ships, the majority of which are pleasure boats constructed from fiberglass that are particularly difficult to detect in SAR imagery. These ships have a very weak SAR signature that is at or below the level of the sea clutter. Furthermore, there are five (5) unassigned SAR detections. After visually inspecting the unassigned SAR detections in the SAR image, it is observed that all five (5) are 'dark' ships that are not transmitting AIS. These ships are located east of the Isle of Wight within the range of terrestrial AIS receiving stations (see Figure 17), and are of a size and predicted class that should be transmitting AIS.

Figure $19 \mathrm{~b}$ shows the distribution of AIS-SAR assignment (or matching) distances. The average distance is $115.6 \mathrm{~m}$ with the majority having a distance less than $100 \mathrm{~m}$.

Table 10. The Solent total number of (top) assignments and unassignments (bottom) features in agreement.

\begin{tabular}{|c|c|c|}
\hline & $S A R_{D}$ & $A I S_{D}$ \\
\hline Total & 26 & 45 \\
\hline Assigned & & \\
\hline$\%$ of Total & 80.8 & 46.7 \\
\hline Unassigned & 5 & 24 \\
\hline$\%$ of Total & 19.2 & 53.3 \\
\hline Assigned & Feature & \# of matches \\
\hline \multirow{3}{*}{21} & Length (valid: 20) & $18(90.0 \%)$ \\
\hline & Width (valid: 20) & $18(90.0 \%)$ \\
\hline & Ship type (valid: 17 ) & $13(76.5 \%)$ \\
\hline
\end{tabular}

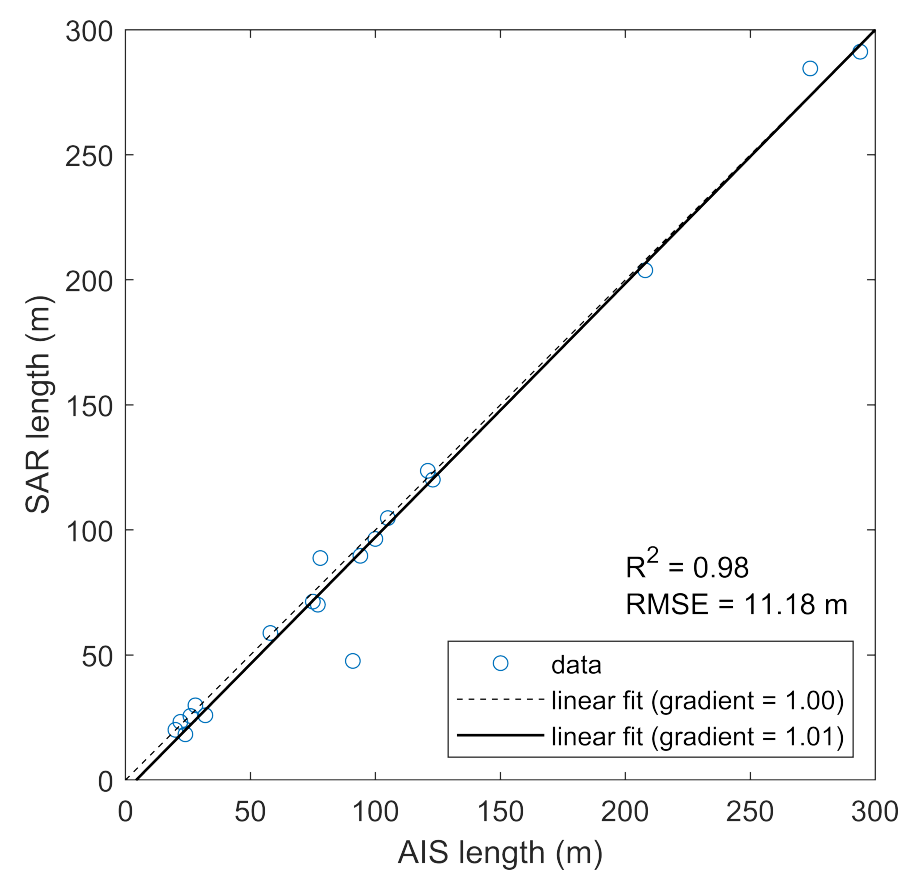

(a)



(b)

Figure 18. The Solent estimated SAR (a) length and (b) width against reported AIS for each assignment. 


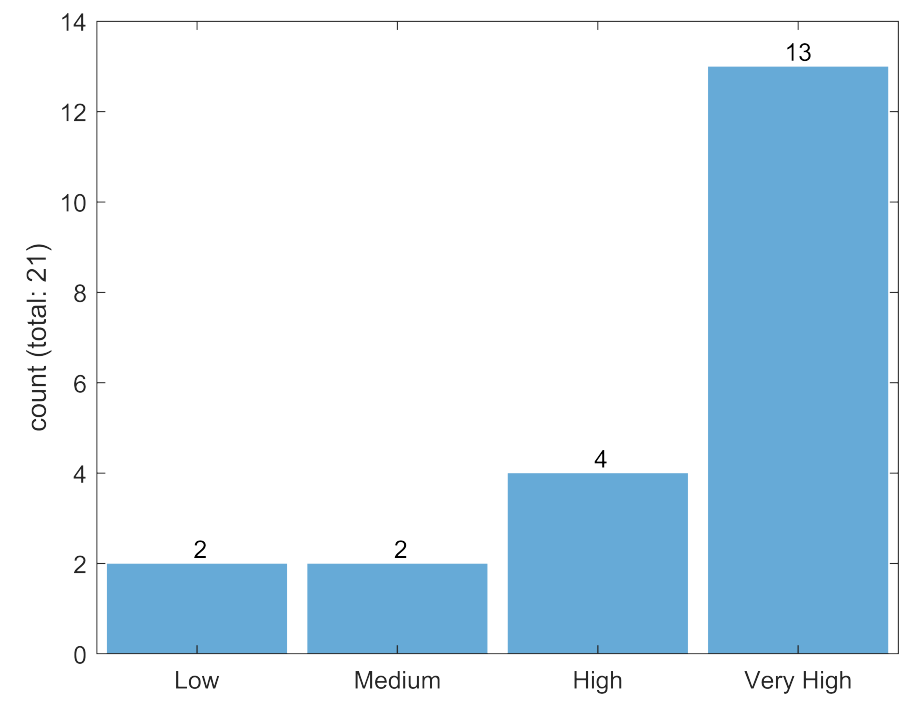

(a)

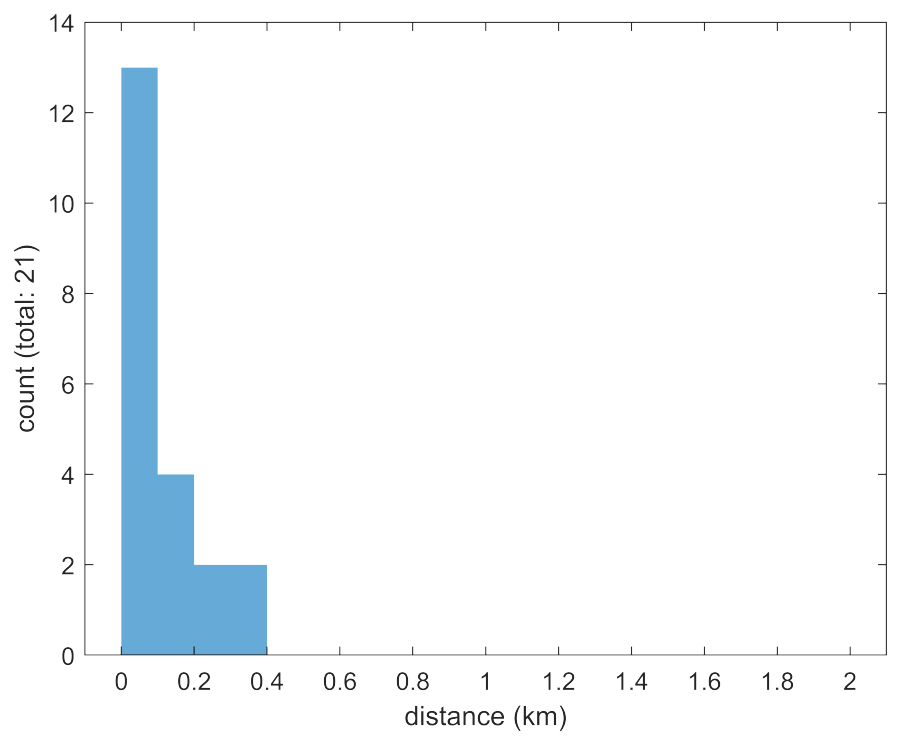

(b)

Figure 19. The Solent (a) AIS-SAR assignment confidence levels and (b) distribution of assignment distances.

\section{Discussion}

\subsection{Effectiveness of Rank-Ordered Assignment}

For Case Study A (English Channel, UK), the results demonstrate a high level of correspondence between the data with $82.6 \%$ of SAR ship detections and $76.4 \%$ of AIS data points being assigned. This is a highly satisfactory result, especially when considering the challenges of wide-area and large-scale data association in very dense shipping environments such as the English Channel. The same SAR product and AIS data are used in the 'English Channel Test Case' in [21], which allows for a broad comparison of the results. For example, in this work, the AIS data processing returns 199 AIS data points compared to the 140 points in [21]. This is thought to be primarily due to the empirical determination of the time interval, $X$. Additionally, AIS-SAR assignments with shorter assignment distances are achieved (see Figure 15b) due to azimuth image shift compensation and accurate interpolation. Typically, it is difficult to carry out azimuth image shift compensation for a large number of ships; however, an automatic and scalable algorithm based on the SAR incidence angle at the AIS-reported position has been used here. Overall, a higher AIS-SAR assignment with good confidence values is achieved.

For Case Study B (The Solent, UK), $80.8 \%$ of SAR ship detections and $46.7 \%$ of AIS data points are assigned. The low number of unassigned SAR ship detections exhibited in both case studies allows for a more efficient investigation into the reasons why detected ships may not be transmitting AIS. The inference of behaviour from 'dark' ships is a compelling research area worth further investigation. Some ships, however, are not detected in the SAR image demonstrating a limitation of the SAR ship detection. The majority of unassigned AIS data points in Case Study B are pleasure boats constructed from fiberglass that are particularly difficult to detect in SAR imagery. Therefore, alternative SAR ship detectors that focus on the detection of small, non-metallic ships may prove to be useful [66]. The fusion workflow (see Figure 1) is highly modular meaning that it is relatively simple to implement alternative SAR ship detectors.

The accurate discrimination of SAR false alarms also has a great effect on the data association performance. For example, in Case Study A (English Channel, UK), the majority of false alarms arise from an imperfect land mask or are caused by coastal infrastructure and azimuth ambiguities. Therefore, improvements in the discrimination of SAR false alarms, such as the use of highly-accurate land masks, will directly improve the overall data association between SAR and AIS. In this work, optical satellite imagery is used to identify fixed structures; however, the use of repeat-pass SAR imagery is an effective 
alternative method to identify specific types of SAR false alarms (e.g., range ambiguities and fixed structures) $[5,19]$.

The rank-ordered assignment algorithm demonstrated its robustness by automatically correcting itself after determining the top-best assignments, corroborating previous simulation results [43]. Figure 20 shows a scenario similar to the conceptual example shown in Figure 6. Initially, the algorithm returns the assignments shown in Figure 20a, which are considered the top-best assignments (i.e., rank equal to one). When considering the geometric and class information, the algorithm correctly updates to the third-best assignments shown in Figure 20b which have better agreement.

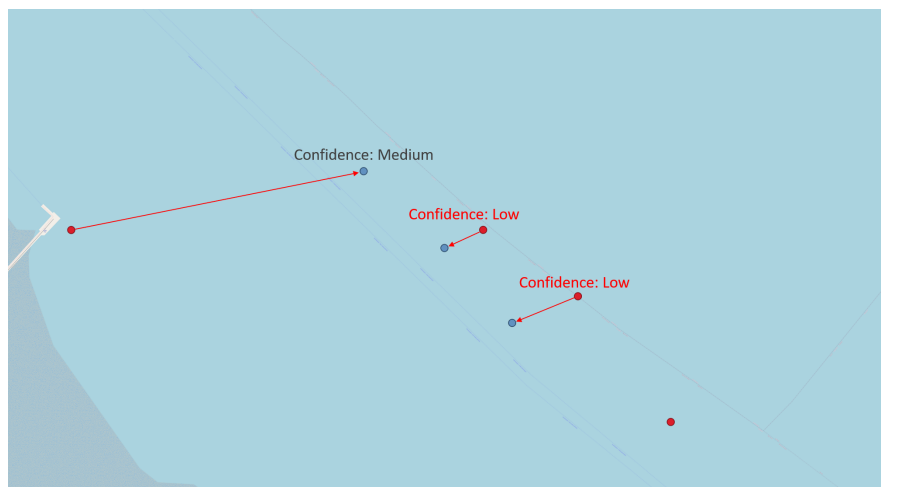

(a)



(b)

Figure 20. Rank-ordered assignment algorithm correctly updated from (a) rank 1 to (b) rank 3 AIS-SAR assignments (AIS data points shown in red and SAR ship detections shown in blue).

\subsection{Effectiveness of Ship Classification}

The SAR ship classification model based on AIS transfer learning shows improvement in the confidence level of the assignments. For Case Study A (English Channel, UK), 25.2\% of the AIS-SAR assignments have a matching ship type, thereby directly improving the confidence level for these assignments. Similarly, 37.7\% received an improved confidence level due to a match in their length and $91.5 \%$ due to a match in their width. In contrast, for Case Study B (the Solent, UK), 76.5\% of the AIS-SAR assignments have a matching ship type. Similarly, $90.0 \%$ received an improved confidence level due to a match in both their length and width.

It is evident that the ship classification model performs better in Case Study B which uses a higher spatial resolution SAR image. This is because more accurate estimates of SAR-derived ship dimensions are obtained leading to better predictions from the model (see Figure 18). The model is also effective over a wider range of ship types (i.e., six classes) for higher spatial resolution compared to previous studies [53,54]. This includes the model correctly predicting the ship classes: cargo, tanker, passenger and tug. For the lower spatial resolution SAR image in Case Study A, an empirical correction has been applied to account for the observed overestimation in ship width returned by the SUMO algorithm with improvements shown (see Figure 14c). However, to improve the estimates of SAR-derived ship dimensions in Sentinel-1 (IW mode) imagery, other more sophisticated SAR ship size estimation techniques are needed (e.g., [63,67]).

As the geometric data are the only transferable features used from AIS, future considerations and potential improvements to the ship classification include finding other transferable features. For example, clustering involves using the location of the vessel in latitude and in longitude as an input feature in order to improve the model's predictive power [60]. Additionally, the use of contextual information involves using the knowledge that the distribution of ship types vary depending on the geographical area. For example, in Southern California, typically a high amount of pleasure craft are observed, whereas, in Southern Louisiana (Gulf of Mexico), a much lower number is observed and tug ships 
make up one of the majority classes. Alongside transferring AIS knowledge, this a priori knowledge can be used to improve SAR ship classification performance.

\section{Conclusions}

A classification-aided data association technique is proposed and evaluated in very dense shipping environments, including the English Channel and The Solent, UK. The research method demonstrates a high level of correspondence between the SAR and AIS data with good confidence values. In particular, the high correlation of SAR ship detections means that it is more practical to investigate 'dark' ships. The SAR ship classification uses a transfer learning method and its performance is evaluated using two SAR products of different spatial resolution. It is shown that implementing ship classification in conjunction with a rank-ordered assignment technique improves the confidence in the data association. The class information helps reduce ambiguity in the rank-ordered assignments, thereby providing a robust match between the data. The transfer learning method shows significant improvement in higher spatial resolution SAR imagery, where the percentage of AISSAR assignments with a match in their ship type increased from $25.2 \%$ in the Sentinel-1 Interferometric Wide (IW) swath mode product to $76.5 \%$ for the ICEYE-X2 Stripmap mode product. Therefore, future work will explore the implementation of more accurate SAR ship size estimation techniques for lower spatial resolution SAR imagery. The data fusion framework described in this paper can also be extended to include other sources of data (e.g., optical, thermal infrared, etc.) which support applications that contribute to maritime safety and security.

Author Contributions: The first author, M.R., was responsible for developing the methodology, implementing the software, validating the results and writing the original draft. The research was supervised by the second author, R.G., who provided regular feedback and reviewed the original draft. All authors have read and agreed to the published version of the manuscript.

Funding: This research was funded by Surrey Satellite Technology Limited (SSTL) as part of a studentship.

Institutional Review Board Statement: Not applicable.

Informed Consent Statement: Not applicable.

Data Availability Statement: The Sentinel-1 SAR product presented in this study is openly available at the Copernicus Open Access Hub.

Acknowledgments: The authors would like to thank LuxSpace Sàrl for freely providing the AIS dataset for the English Channel case study. They would also like to thank Spire Maritime for freely providing the AIS dataset for the Solent case study.

Conflicts of Interest: The authors declare no conflict of interest.

\section{References}

1. ITU Radiocommunication Sector (ITU-R). Technical Characteristics for An Automatic Identification System Using Time-Division Multiple Access in The Vhf Maritime Mobile Band; Recommendation ITU-R M.1371-5; International Telecommunication Union (ITU): Geneva, Switzerland, 2014.

2. Brusch, S.; Lehner, S.; Fritz, T.; Soccorsi, M.; Soloviev, A.; van Schie, B. Ship Surveillance With TerraSAR-X. IEEE Trans. Geosci. Remote Sens. 2011, 49, 1092-1103. [CrossRef]

3. Vachon, P.W.; Kabatoff, C.; Quinn, R. Operational ship detection in Canada using RADARSAT. In Proceedings of the 2014 IEEE Geoscience and Remote Sensing Symposium, Quebec City, QC, Canada, 13-18 July 2014; pp. 998-1001. [CrossRef]

4. Velotto, D.; Bentes, C.; Tings, B.; Lehner, S. First, Comparison of Sentinel-1 and TerraSAR-X Data in the Framework of Maritime Targets Detection: South Italy Case. IEEE J. Ocean. Eng. 2016, 41, 993-1006. [CrossRef]

5. Santamaria, C.; Alvarez, M.; Greidanus, H.; Syrris, V.; Soille, P.; Argentieri, P. Mass Processing of Sentinel-1 Images for Maritime Surveillance. Remote Sens. 2017, 9, 678. [CrossRef]

6. Lehner, S.; Brusch, S.; Fritz, T. Ship surveillance by joint use of SAR and AIS. In Proceedings of the OCEANS 2009-EUROPE, Bremen, Germany, 11-14 May 2009; pp. 1-5. [CrossRef] 
7. Posada, M.; Greidanus, H.; Alvarez, M.; Vespe, M.; Cokacar, T.; Falchetti, S. Maritime awareness for counter-piracy in the Gulf of Aden. In Proceedings of the 2011 IEEE International Geoscience and Remote Sensing Symposium, Vancouver, BC, Canada, 24-29 July 2011; pp. 249-252. [CrossRef]

8. $\quad$ Longépé, N.; Hajduch, G.; Ardianto, R.; de Joux, R.; Nhunfat, B.; Marzuki, M.I.; Fablet, R.; Hermawan, I.; Germain, O.; Subki, B.A.; et al. Completing fishing monitoring with spaceborne Vessel Detection System (VDS) and Automatic Identification System (AIS) to assess illegal fishing in Indonesia. Mar. Pollut. Bull. 2018, 131, 33-39. [CrossRef] [PubMed]

9. Rowlands, G.; Brown, J.; Soule, B.; Boluda, P.T.; Rogers, A.D. Satellite surveillance of fishing vessel activity in the Ascension Island Exclusive Economic Zone and Marine Protected Area. Mar. Policy 2019, 101, 39-50. [CrossRef]

10. Kurekin, A.A.; Loveday, B.R.; Clements, O.; Quartly, G.D.; Miller, P.I.; Wiafe, G.; Adu Agyekum, K. Operational Monitoring of Illegal Fishing in Ghana through Exploitation of Satellite Earth Observation and AIS Data. Remote Sens. 2019, 11, 293. [CrossRef]

11. Park, J.; Lee, J.; Seto, K.; Hochberg, T.; Wong, B.A.; Miller, N.A.; Takasaki, K.; Kubota, H.; Oozeki, Y.; Doshi, S.; et al. Illuminating dark fishing fleets in North Korea. Sci. Adv. 2020, 6. [CrossRef]

12. Uiboupin, R.; Raudsepp, U.; Sipelgas, L. Detection of oil spills on SAR images, identification of polluters and forecast of the slicks trajectory. In Proceedings of the 2008 IEEE/OES US/EU-Baltic International Symposium, Tallinn, Estonia, 27-29 May 2008; pp. 1-5. [CrossRef]

13. Longépé, N.; Mouche, A.; Goacolou, M.; Granier, N.; Carrere, L.; Lebras, J.; Lozach, P.; Besnard, S. Polluter identification with spaceborne radar imagery, AIS and forward drift modeling. Mar. Pollut. Bull. 2015, 101, 826-833. [CrossRef]

14. Garello, R.; Kerbaol, V. Oil pollution monitoring: An integrated approach. In Proceedings of the 2017 IEEE Workshop on Environmental, Energy, and Structural Monitoring Systems (EESMS), Milan, Italy, 24-25 July 2017; pp. 1-6. [CrossRef]

15. English, J.; Hewitt, R.; Power, D.; Tunaley, J. ICE-SAIS - Space-based AIS and SAR for improved Ship and Iceberg Monitoring. In Proceedings of the 2013 IEEE Radar Conference (RadarCon13), Ottawa, ON, Canada, 29 April-3 May 2013; pp. 1-6. [CrossRef]

16. Marino, A.; Hajnsek, I. Statistical Tests for a Ship Detector Based on the Polarimetric Notch Filter. IEEE Trans. Geosci. Remote Sens. 2015, 53, 4578-4595. [CrossRef]

17. Touzi, R.; Vachon, P.W. RCM Polarimetric SAR for Enhanced Ship Detection and Classification. Can. J. Remote Sens. 2015, 41, 473-484. [CrossRef]

18. Iervolino, P.; Guida, R. A Novel Ship Detector Based on the Generalized-Likelihood Ratio Test for SAR Imagery. IEEE J. Sel. Top. Appl. Earth Obs. Remote Sens. 2017, 10, 3616-3630. [CrossRef]

19. Greidanus, H.; Alvarez, M.; Santamaria, C.; Thoorens, F.X.; Kourti, N.; Argentieri, P. The SUMO Ship Detector Algorithm for Satellite Radar Images. Remote Sens. 2017, 9, 246. [CrossRef]

20. Sandirasegaram, N.; Vachon, P.W. Validating Targets Detected by SAR Ship Detection Engines. Can. J. Remote Sens. 2017, 43, 451-454. [CrossRef]

21. Pelich, R.; Chini, M.; Hostache, R.; Matgen, P.; Lopez-Martinez, C.; Nuevo, M.; Ries, P.; Eiden, G. Large-Scale Automatic Vessel Monitoring Based on Dual-Polarization Sentinel-1 and AIS Data. Remote Sens. 2019, 11, 1078. [CrossRef]

22. Margarit, G.; Barba Milanés, J.A.; Tabasco, A. Operational Ship Monitoring System Based on Synthetic Aperture Radar Processing. Remote Sens. 2009, 1, 375-392. [CrossRef]

23. Margarit, G.; Tabasco, A. Ship Classification in Single-Pol SAR Images Based on Fuzzy Logic. IEEE Trans. Geosci. Remote Sens. 2011, 49, 3129-3138. [CrossRef]

24. Xing, X.; Ji, K.; Zou, H.; Chen, W.; Sun, J. Ship Classification in TerraSAR-X Images With Feature Space Based Sparse Representation. IEEE Geosci. Remote Sens. Lett. 2013, 10, 1562-1566. [CrossRef]

25. Wang, C.; Zhang, H.; Wu, F.; Jiang, S.; Zhang, B.; Tang, Y. A Novel Hierarchical Ship Classifier for COSMO-SkyMed SAR Data. IEEE Geosci. Remote Sens. Lett. 2014, 11, 484-488. [CrossRef]

26. Fernandez Arguedas, V.; Velotto, D.; Tings, B.; Greidanus, H.; Bentes da Silva, C.A. Ship classification in high and very high resolution satellite SAR imagery. In Proceedings of the Security Research Conference, 11th Future Security, Berlin, Germany, 13-14 September 2016; pp. 347-354.

27. Jiang, M.; Yang, X.; Dong, Z.; Fang, S.; Meng, J. Ship Classification Based on Superstructure Scattering Features in SAR Images. IEEE Geosci. Remote Sens. Lett. 2016, 13, 616-620. [CrossRef]

28. Huang, L.; Liu, B.; Li, B.; Guo, W.; Yu, W.; Zhang, Z.; Yu, W. OpenSARShip: A Dataset Dedicated to Sentinel-1 Ship Interpretation. IEEE J. Sel. Top. Appl. Earth Obs. Remote Sens. 2018, 11, 195-208. [CrossRef]

29. Hou, X.; Ao, W.; Song, Q.; Lai, J.; Wang, H.; Xu, F. FUSAR-Ship: Building a high-resolution SAR-AIS matchup dataset of Gaofen-3 for ship detection and recognition. Sci. China Inf. Sci. 2020, 63. [CrossRef]

30. Song, J.; Kim, D.J.; Kang, K.M. Automated Procurement of Training Data for Machine Learning Algorithm on Ship Detection Using AIS Information. Remote Sens. 2020, 12, 1443. [CrossRef]

31. Grasso, R.; Mirra, S.; Baldacci, A.; Horstmann, J.; Coffin, M.; Jarvis, M. Performance Assessment of a Mathematical Morphology Ship Detection Algorithm for SAR Images through Comparison with AIS Data. In Proceedings of the 2009 Ninth International Conference on Intelligent Systems Design and Applications, Pisa, Italy, 30 November-2 December 2009; pp. 602-607. [CrossRef]

32. Gurgel, K.; Schlick, T.; Horstmann, J.; Maresca, S. Evaluation of an HF-radar ship detection and tracking algorithm by comparison to AIS and SAR data. In Proceedings of the 2010 International WaterSide Security Conference, Carrara, Italy, 3-5 November 2010; pp. 1-6. [CrossRef] 
33. Chaturvedi, S.K.; Yang, C.S.; Ouchi, K.; Shanmugam, P. Ship Recognition by Integration of SAR and AIS. J. Navig. 2012, 65, 323-337. [CrossRef]

34. Mazzarella, F.; Alessandrini, A.; Greidanus, H.; Alvarez, M.; Argentieri, P.; Nappo, D.; Ziemba, L. Data fusion for wide-area maritime surveillance. In Proceedings of the COST MOVE Workshop on Moving Objects at Sea, Brest, France, 27-28 June 2013, pp. 27-28.

35. Voinov, S.; Schwarz, E.; Krause, D.; Berg, M. Identification of SAR Detected Targets on Sea in Near Real Time Applications for Maritime Surveillance. In Proceedings of the Free and Open Source Software for Geospatial (FOSS4G) Conference, Bonn, Germany, 24-26 August 2016; Volume 16. [CrossRef]

36. Mazzarella, F.; Vespe, M.; Santamaria, C. SAR Ship Detection and Self-Reporting Data Fusion Based on Traffic Knowledge. IEEE Geosci. Remote Sens. Lett. 2015, 12, 1685-1689. [CrossRef]

37. Zhang, H.; Liu, Y.; Ji, Y.; Wang, L.; Zhang, J. Multi-Feature Maximum Likelihood Association with Space-borne SAR, HFSWR and AIS. J. Navig. 2017, 70, 359-378. [CrossRef]

38. Blackman, S.S.; Popoli, R. Design and Analysis of Modern Tracking Systems; Artech House: Norwood, MA, USA, 1999.

39. Bar-Shalom, Y.; Blair, W.D. Multitarget-Multisensor Tracking: Applications and Advances; Artech House: Norwood, MA, USA, 2000; Volume 3.

40. Zhao, Z.; Ji, K.; Xing, X.; Zou, H.; Zhou, S. Ship Surveillance by Integration of Space-borne SAR and AIS - Further Research. J. Navig. 2014, 67, 295-309. [CrossRef]

41. Zhao, Z.; Ji, K.F.; Xing, X.W.; Zou, H.X. Effective Association of SAR and AIS Data Using Non-Rigid Point Pattern Matching. IOP Conf. Ser. Earth Environ. Sci. 2014, 17, 012258. [CrossRef]

42. Liu, Y.; Yao, L.; Xiong, W.; Zhou, Z. GF-4 Satellite and Automatic Identification System Data Fusion for Ship Tracking. IEEE Geosci. Remote Sens. Lett. 2019, 16, 281-285. [CrossRef]

43. Rodger, M.; Guida, R. Data Association Techniques for Near-Contemporaneous SAR and AIS Datasets from NovaSAR-1. In Proceedings of the IGARSS 2019-2019 IEEE International Geoscience and Remote Sensing Symposium, Yokohama, Japan, 28 July-2 August 2019; pp. 700-703. [CrossRef]

44. Vachon, P.W.; English, R.A.; Wolfe, J. Validation of RADARSAT-1 vessel signatures with AISLive data. Can. J. Remote Sens. 2007, 33, 20-26. [CrossRef]

45. Surrey Satellite Technology Limited (SSTL). NovaSAR-1: Launched 2018. Available online: https://www.sstl.co.uk/spaceportfolio/launched-missions/2010-2018/novasar-1 (accessed on 26 October 2020).

46. EoPortal. PAZ SAR Satellite Mission of Spain. Available online: https://directory.eoportal.org/web/eoportal/satellite-missions / $\mathrm{p} / \mathrm{paz}$ (accessed on 26 October 2020).

47. Canadian Space Agency. RADARSAT Constellation Mission. Available online: http://www.asc-csa.gc.ca/eng/satellites/ radarsat/default.asp (accessed on 26 October 2020).

48. Raney, R.K. Synthetic Aperture Imaging Radar and Moving Targets. IEEE Trans. Aerosp. Electron. Syst. 1971, AES-7, 499-505. [CrossRef]

49. Ouchi, K. On the multilook images of moving targets by synthetic aperture radars. IEEE Trans. Antennas Propag. 1985, 33, 823-827. [CrossRef]

50. Lin, I.I.; Khoo, V. Computer-based algorithm for ship detection from ERS SAR imagery. In Proceedings of the Third ERS Symposium on Space at the service of our Environment, Florence, Italy, 14-21 March 1997; Volume 414, pp. 1411-1416.

51. Ouchi, K.; Iehara, M.; Morimura, K.; Kumano, S.; Takami, I. Nonuniform azimuth image shift observed in the Radarsat images of ships in motion. IEEE Trans. Geosci. Remote Sens. 2002, 40, 2188-2195. [CrossRef]

52. Vachon, P.; English, R.; Wolfe, J. Ship Signatures in RADARSAT-1 ScanSAR Narrow B Imagery: Analysis with AISLive Data; Technical Report; Defence Research and Development Canada (DRDC): Ottawa, ON, Canada, 2007.

53. Lang, H.; Wu, S.; Xu, Y. Ship Classification in SAR Images Improved by AIS Knowledge Transfer. IEEE Geosci. Remote Sens. Lett. 2018, 15, 439-443. [CrossRef]

54. Snapir, B.; Waine, T.W.; Biermann, L. Maritime Vessel Classification to Monitor Fisheries with SAR: Demonstration in the North Sea. Remote Sens. 2019, 11, 353. [CrossRef]

55. Murty, K.G. An Algorithm for Ranking all the Assignments in Order of Increasing Cost. Oper. Res. 1968, 16, 682-687. [CrossRef]

56. Jonker, R.; Volgenant, A. A shortest augmenting path algorithm for dense and sparse linear assignment problems. Computing 1987, 38, 325-340. [CrossRef]

57. Greidanus, H.; Argentieri, P.; Alvarez, M.; Santamaria, C.; Kourti, N. The SUMO Ship Detection Software for Satellite Radar Images_Short Installation and User Guide; Technical Report; Joint Research Centre (JRC): Ispra, Italy, 2017.

58. Last, P.; Bahlke, C.; Hering-Bertram, M.; Linsen, L. Comprehensive Analysis of Automatic Identification System (AIS) Data in Regard to Vessel Movement Prediction. J. Navig. 2014, 67, 791-809. [CrossRef]

59. ShipAIS. Available online: http://www.shipais.com/(accessed on 26 October 2020).

60. Rodger, M.; Guida, R. SAR and AIS Data Fusion for Dense Shipping Environments. In Proceedings of the IGARSS 2020 -2020 IEEE International Geoscience and Remote Sensing Symposium, Virtual Symposium, 26 September-2 October 2020.

61. Seiffert, C.; Khoshgoftaar, T.M.; Van Hulse, J.; Napolitano, A. RUSBoost: Improving classification performance when training data are skewed. In Proceedings of the 2008 19th International Conference on Pattern Recognition, Tampa, FL, USA, 8-11 December 2008; pp. 1-4. [CrossRef] 
62. Chawla, N.V.; Lazarevic, A.; Hall, L.O.; Bowyer, K.W. SMOTEBoost: Improving Prediction of the Minority Class in Boosting. In Knowledge Discovery in Databases: PKDD 2003; Lavrač, N., Gamberger, D., Todorovski, L., Blockeel, H., Eds.; Springer: Berlin/Heidelberg, Germany, 2003; pp. 107-119.

63. Stasolla, M.; Greidanus, H. The exploitation of Sentinel-1 images for vessel size estimation. Remote Sens. Lett. 2016, 7, 1219-1228. [CrossRef]

64. Greidanus, H. Sub-aperture Behavior of SAR Signatures of Ships. In Proceedings of the 2006 IEEE International Symposium on Geoscience and Remote Sensing, Denver, CO, USA, 31 July-4 August 2006; pp. 3579-3582. [CrossRef]

65. Schubert, A.; Miranda, N.; Geudtner, D.; Small, D. Sentinel-1A/B Combined Product Geolocation Accuracy. Remote Sens. 2017, 9, 607. [CrossRef]

66. Liu, G.; Zhang, X.; Meng, J. A Small Ship Target Detection Method Based on Polarimetric SAR. Remote Sens. 2019, 11, 2938. [CrossRef]

67. Li, B.; Liu, B.; Guo, W.; Zhang, Z.; Yu, W. Ship Size Extraction for Sentinel-1 Images Based on Dual-Polarization Fusion and Nonlinear Regression: Push Error Under One Pixel. IEEE Trans. Geosci. Remote Sens. 2018, 56, 4887-4905. [CrossRef] 